\title{
Circuit
}

Musiques contemporaines

\section{Qu'est-ce concert?}

\section{Robert Normandeau}

Volume 13, numéro 1, 2002

L'électroacoustique : à la croisée des chemins?

URI : https://id.erudit.org/iderudit/902264ar

DOI : https://doi.org/10.7202/902264ar

Aller au sommaire du numéro

\section{Éditeur(s)}

Les Presses de l'Université de Montréal

\section{ISSN}

1183-1693 (imprimé)

1488-9692 (numérique)

Découvrir la revue

\section{Citer cet article}

Normandeau, R. (2002). Qu'est-ce concert? Circuit, 13(1), 43-50. https://doi.org/10.7202/902264ar

\section{Résumé de l'article}

« Le concert : pourquoi? Comment? " titrait une publication de l'INA-GRM parue en 1977. Où en sommes-nous vingt-cinq ans plus tard dans ce mode de représentation de la musique acousmatique? Que peut-on répondre en effet à ceux qui remettent en question non seulement la façon de présenter la musique de support en concert mais la pertinence même de ce genre de concert? Que de fois n'avons-nous pas entendu qu'il est totalement inutile de s'asseoir devant des haut-parleurs où il n’y a « rien à voir "! (référence à la série de concerts exclusivement dédiée à la musique acousmatique produite à Montréal). Dans cet article, l'auteur tente de répondre à ces questions en s'attardant au concept de rituel et à la place occupée par l'interprétation dans les concerts acoustiques.
Ce document est protégé par la loi sur le droit d'auteur. L'utilisation des services d'Érudit (y compris la reproduction) est assujettie à sa politique d'utilisation que vous pouvez consulter en ligne.

https://apropos.erudit.org/fr/usagers/politique-dutilisation/ 


\title{
Qu'est-ce concert' ?
}

\author{
Robert Normandeau
}

"Qu'est-ce qu'on sert ce soir?", cette question silencieuse, il se trouve (encore) des mélomanes pour venir dans des salles la poser à un mur - le mur des haut-parleurs qui ne sait, apparemment, que leur en renvoyer l'écho, sous la forme inversée d'un vacarme acousmatique.

Mur enveloppant dans certains cas, répercutant cet écho en faisceaux multiples, mais n'en renvoyant pas moins la question dans la salle, puisque sur scène nul n'est là pour s'en faire, par ses gestes et sa mimique d'instrumentiste, le répondant. Cette communication publique, mais seul à seuls, d'un chacun avec les sons, c'est cette forme spéciale, austère, controversée, mais fascinante quand elle fonctionne, de concert qu'on appelle vulgairement "de bandes » ou de "haut-parleurs» ou "électroacoustique», ou enfin "acousmatique " plus noblement.

Michel Chion

"Le concert, pourquoi? Comment $?^{2}$ » titrait déjà une publication de l'INA-GRM parue en 1977 d'où est tirée cette citation. Où en sommes-nous, vingt-cinq ans plus tard, dans ce mode de représentation de la musique acousmatique? Que peuton répondre, en effet, à ceux qui remettent en question non seulement la façon de présenter la musique de support en concert mais la pertinence même de ce genre de concert? Que de fois n'avons-nous pas entendu qu'il est totalement inutile de s'asseoir devant des haut-parleurs où il n'y a "rien à voir " " Depuis l'apparition du disque compact au début des années 1980, cette musique n'a-telle pas trouvé son mode de diffusion idéal ẹ Et puis ce compositeur présent devant la console de diffusion joue-til vraiment un rôle d'interprète ou n'estil là qu'un vestige de l'interprète d'autrefois, mimant un simulacre de concert traditionnel ? On le voit, les questions sont nombreuses et les réponses sans doute pas aussi évidentes à formuler qu'on le croirait, le concert d'aujourd'hui ressemblant à s'y méprendre à celui auquel assistait les auditeurs de Beethoven, même lors des manifestations musicales les plus contemporaines qui soient.
1. Titre d'une pièce de Mario Rodrigue publié en 1987 sur le disque Alchimie (empreintes DIGITALes, IMED9415)

2. «le concert, pourquoi? Comment? », Cahiers Recherche/Musique 5 (1977), Paris, INA-GRM.

3. Titre d'une série de concerts produite à Montréal par Réseaux depuis 1997 et exclusivement dédiée à la musique acousmatique. 


\section{Le concert}

Sur le plan historique,

«le concert ne se définit avec précision que s'il existe un auditoire spécialement réuni en vue d'écouter un programme prévu à l'avance. Dans cette optique, ses origines se perdent dans la nuit des temps. Aussi bien se limitet-on ici à l'Occident et au sens restreint qui a été donné au terme, généralement dans un cadre urbain et sur une base commerciale. Avant le XVIle siècle, la musique s'insérait naturellement dans le cadre de la vie quotidienne. Aussi, est-on frappé par l'originalité des auditions prévues par les statuts de l'Académie de poésie et de musique de Jean-Antoine de Baiff et Thibault de Courville (1570) : deux heures chaque dimanche devant un public payant. C'est vers le milieu du XVII e siècle que prend forme, au moins en France, en Italie et en Angleterre, un certain type de concerts plus ou moins réguliers et ouverts au public ${ }^{4}$.

Mais c'est véritablement au XIXe siècle que s'établira la forme des concerts telle 4. Dictionnaire de la musique, «Techniques formes Instruments (1976), Paris, Bordas.

\section{Projeter en commun}

De l'étymologie du mot, je retiens les origines suivantes : concerter 1437, Chartier, "projeter en commun"; 1665 ital. concerto, accord du lat. concertus, concerté . $^{2}$.

Comment le concert fonctionne-til ? Quels sont les mécanismes qui le régissent? Pourquoi des dizaines, des centaines voire des milliers d'individus se réunissentils dans un même lieu pour assister à un concert? J'allais écrire, pour "voir» un concert! N'y a-til pas là un premier élément de réponse? En effet, l'auditeur se projette psychologiquement dans l'interprète qui est devant lui. II est l'interprète. C'est lui qui monte sur la scène, qu'on acclame ou qu'on persifle, c'est selon, cela faisant aussi partie du rituel. On est au cirque, au spectacle. La diva qui craque son contre-ut, le pianiste qui plaque un accord dissonant, le guitariste de rock qui casse une corde, le saxophoniste de jazz qui n'arrive plus à rien extirper des vapeurs illicites, tout cela contribue à créer une tension qui tient le public en haleine. En payant sa place, le spectateur délègue à l'artiste un rôle symbolique qui permet à ce dernier de canaliser la magie et le mystère qui entoure la musique, surtout pour ceux qui ne la pratiquent pas, mais également pour ceux qui ne la pratiquent pas à ce niveau. Tout le monde a déjà un peu tâté du ballon de soccer,
5. Dictionnaire étymologique, (2002), Paris, Larousse. 
mais personne ne joue comme Pele ${ }^{6}$. Alors sur le terrain, il nous représente, il magnifie nos propres aptitudes comme le musicien sur scène qui fait mieux que nous tous ce que nous imaginons possible. Le musicien de scène nous projette en commun. Nous allons au concert afin qu'il prenne en charge nos émotions et nous emmène là où nous ne pouvons nous rendre seuls, soit que nous n'en ayons pas la capacité technique, soit parce que le phénomène du rituel induit des comportements inaccessibles autrement, particulièrement dans le confort de son foyer.
6. Edson Arantes do Nascimento (1940Brésil), célèbre joueur de soccer (football) brésilien des années 1960 considéré comme le plus grand joueur de tous les temps.

\section{Le lieu du rituel}

La forme actuelle du concert est demeurée pratiquement inchangée depuis la fin du $X V I I I{ }^{e}$ siècle, qu'il s'agisse de musique classique, contemporaine ou populaire. Même les manifestations les plus "avant-gardistes" en musique électronique reprennent le modèle traditionnel constitué d'une scène sur laquelle l'artiste officie et d'un public qui vient le voir. Le fait de pouvoir circuler dans la salle de concert en prenant une bière et en discutant avec son voisin n'est jamais que la prolongation des pratiques ancestrales du théâtre, où le parterre était réservé au peuple qui y devisait en toute liberté, au grand dam des artistes sur scène. La question peut alors se poser en ces termes : la forme du concert telle qu'elle s'est développée à travers les âges seraitelle parvenue à un état de développement idéal, tellement que personne ne songerait plus à la remettre en question? Ce qui se concrétiserait par la salle à l'italienne et ses multiples variantes. Ou alors peut-être s'agitil simplement d'une longue habitude qui, comme la forme de l'orchestre classique, est demeurée figée dans le temps, incapable de se renouveler et entrant par le fait même dans une certaine décadence? Le lieu du rituel mérite pourtant d'être questionné, comme tous les aspects reliés au concert. D'autant plus lorsqu'il s'agit d'une musique qui est aussi radicale dans son mode de présentation que l'acousmatique.
7. On pense à Elektra ou à Mutek à Montréal, par exemple.

\section{Le rituel lui-même}

Assister à un concert, c'est convenir que l'on va partager avec d'autres auditeurs le même espace-temps. On vient là parce qu'on a envie d'entendre l'artiste en question mais aussi parce qu'on a envie de l'entendre dans ces conditions-là. Cela est d'autant plus vrai que la plupart des rituels sociaux, dans les sociétés occidentales tout au moins, ont disparu. On ne va plus à l'église, on ne se réunit plus guère pour les fêtes de villages et de toute manière il n'y a plus de villages 
puisque plus de $90 \%$ des Occidentaux vivent en ville aujourd'hui. Mais il semble bien que, malgré cela, le rituel soit nécessaire, voire même vital pour l'être humain, qui est essentiellement, rappelons-le, un animal social. Donc, on va au concert comme on allait à l'église autrefois. Les deux modes ont cohabité longtemps d'ailleurs et le concert classique tout au moins a conservé tous les attributs de l'office liturgique : sobriété de l'officiant (sauf pour les divas d'aujourd'hui... qui rivalisent toutes d'imagination dans le kitsch de leurs accoutrements!), respect des spectateurs devant celui-ci, respect des conventions, etc. La seule chose qui n'est pas empruntée à l'office religieux au concert, ce sont les applaudissements, qui proviennent plutôt des jeux et du cirque. Le rituel du concert est donc un mélange entre l'office et le cirque.

\section{Qu'est-ce qu'on sert?}

Pour paraphraser le jeu de mot du titre de Rodrigue qui coiffe cet article, mais qu'est-ce qui nous est donc offert, comme auditeur, par les producteurs de concerts acousmatiques, qu'est-ce qu'on nous sert? Sur le plan du rituel, on peut constater que le concert traditionnel a bien laissé ça et là quelques traces évidentes : il y a bien là des sièges, un public, une scène, généralement vide. Et il y a bien un officiant qui se présente dans la salle mais qui prendra place, plus souvent qu'autrement, au milieu du public. Et c'est là que tout bascule. Le concert de musique acousmatique ressemble à s'y méprendre au concert traditionnel mais on se rend compte tout d'un coup qu'il y manque un élément essentiel : l'occupation de la scène. L'artiste est bien là, mais il est parmi nous! Et parce qu'il est si proche, parce que nous pouvons le toucher, il devient comme nous, non pas en tant que délégué de nos aspirations les plus profondes, mais plutôt comme un participant ordinaire. Et ce faisant, il dévalue considérablement son pouvoir de projection et de cristallisation de nos aspirations, d'autant plus que nous ne pouvons absolument pas nous projeter dans ses gestes puisque nous en ignorons, pour la majorité d'entre nous tout au moins, le sens. Et cela est d'autant plus vrai que ceuxci sont généralement discrets, voire effacés. D'où l'échec relatif de la grande majorité des concerts acousmatiques depuis que ceux-ci sont passés de mode ${ }^{8}$ : le public ne s'y reconnaît pas ou au contraire, s'y reconnaît trop! Rien de pire pour accroître ce sentiment terre à terre, que des techniciens qui s'affairent entre les pièces! Comment aspirer à un dépassement de soi quand on nous présente toute la cuisine derrière le rituel? C'est comme si on venait laver la vaisselle devant nous entre chaque service d'un repas au restaurant... Sur ce plan, les organisateurs de concerts acousmatiques ont certainement un examen de conscience à faire.
8. Rappelons pour mémoire que le concert acousmatique n'a pas toujours connu la défaveur du public : dans les années 1960 et 1970, il n'était pas rare qu'on remplisse des salles plusieurs soirs de suite avec le même programme ou même qu'on présente des concerts dans des stades. Il faut cependant ajouter qu'en général ces concerts comportaient aussi des éléments spectaculaires : lasers, lumières, costumes, etc. 


\section{Et pourtant! Un : le rituel}

Le concert demeure aujourd'hui le dernier espace de rituel pour l'écoute de la musique. En effet, qui prend aujourd'hui le temps d'écouter de la musique? Je veux dire prendre le temps de ne faire que cela : écouter de la musique? Se poser la question et la poser autour de soi, c'est y répondre : pratiquement personne, pratiquement jamais. Et tous styles confondus. Là où autrefois, on se réunissait pour jouer en formation de chambre ou pour écouter cette musique ; là où nos parents et nos grands-parents se réunissaient pour écouter $L^{\prime} O p e ́ r a ~ d u ~ s a m e d i^{10}$; là n'existe plus aujourd'hui que la radio guimauve des salles d'attente et des lieux publics; là ne subsiste que des musiques destinées à accompagner les activités du quotidien : musique pour le dîner, musique pour la vaisselle, etc. Ne reste plus dans la sphère du privé que l'écoute praticienne, celle du spécialiste qui écoute afin de remplir la programmation de son prochain festival!

Et pourtant, prendre la décision de se rendre dans un lieu, à une date et à une heure déterminées dans le but de partager un même temps avec d'autres individus demeure encore, et de loin, la meilleure manière d'accéder à la vérité de l'œuvre et partant de découvrir en soi ce qu'il y a d'universel dans toute proposition artistique. La qualité de la concentration induite par le rituel grâce au temps partagé avec d'autres auditeurs permet une qualité de l'écoute exceptionnelle (par le fait qu'il n'y a pas le choix : pas moyen de mettre sur pause pour aller se chercher une bière ou de zapper...). Et cela est d'autant plus vrai lorsqu'il n'y a "rien à voir». Cette situation apparaît certes paradoxale dans notre société de l'image et du divertissement et il se peut fort bien que le rituel soit sur un déclin irrémédiable. Bien téméraire serait celui qui l'affirmerait à coup sûr, mais les statistiques des cinquante dernières années sur la fréquentation à la baisse des salles de concert le conforteraient certainement dans son affirmation. Mais le phénomène n'est pas que musical : le cinéma et le théâtre vivent la même situation. II s'agit d'un phénomène de société qui dépasse le cadre spécifique de la musique et plus encore celui de la musique acousmatique.
9. Rappelons qu'avant la Seconde Guerre mondiale, donc avant l'apparition de la haute-fidélité, il n'existait que deux façons d'écouter la musique : la faire ou se rendre à la salle de concert. La radio d'abord et le disque ensuite feront disparaître progressivement ces attitudes actives au profit de comportements beaucoup plus passits.

10. Un rendez-vous radiophonique toujours présent en 2002 à la chaîne culturelle de la radio de Radio-Canada!

\section{Et pourtant! Deux : I'interprétation}

Il y a deux ans, j'ai eu l'occasion de participer comme membre du jury, au premier concours international de spatialisation Métamorphoses d'Orphée "l. II s'agissait en quelque sorte, à travers ce concours, de valider l'idée selon laquelle la musique acousmatique gagne (et parfois perd...) à être interprétée en concert. Et de tenter de déterminer, à travers les discussions du jury qui faisaient suite aux différentes
11. Organisé par Annette Vande Gorne à Bruxelles. 
interprétations d'une même œuvre par les candidats, ce qui en faisait la spécificité. Je ne suis pas certain que nous sommes parvenus à définir très clairement ce qui était de l'ordre de l'universel et du particulier - qu'est-ce qui appartient en propre à une œuvre et qui doit être intégralement respecté et qu'est-ce qui appartient à la sphère de l'interprétation et est donc variable comme les interprètes différents qui se succèdent à la console - mais ce qui est certain, c'est que nous avons entendu très clairement des différences et que nous avons réussi à statuer, et de façon unanime, sur le premier prix du concours.

$\|$ y a donc une part d'interprétation dans cette musique, pourtant "fixée ${ }^{12}$ » sur un support et invariable, sur le plan temporel tout au moins, d'une écoute à l'autre. Et cette part d'interprétation n'est pas rien, elle est même, nous en sommes intimement convaincu, une part aussi importante que l'œuvre elle-même lorsque celle-ci est présentée en concert. Certaines œuvres, d'ailleurs, ne vivent que très difficilement en dehors de la salle de concert, comme certains films survivent difficilement au passage vers le petit écran, auquel l'écoute sur disque peut se comparer. Or, si cette interprétation est tellement essentielle, comment se fait-il que dans les faits, presque tous les organisateurs de concerts n'y consacrent que très peu de temps? $\|$ est d'usage de considérer qu'une heure de répétition avant un concert constitue une durée raisonnable. En cela, on reprend encore une fois le modèle du concert classique qui stipule que l'interprète connaît l'œuvre à l'avance et qu'il n'a besoin que de quelques instants pour se familiariser avec l'instrument et la salle. Or dans le cas du concert acousmatique, le problème, c'est que l'instrument n'existe pas en dehors de cette salle de concert, pour ce concert particulier! Car l'interprétation de cette musique est bien de l'ordre de la mise en espace. Or cet espace est unique et il faut du temps pour l'apprivoiser, pour se le mettre dans les doigts, pour emprunter une expression propre au langage instrumental.
12. D'après Michel Chion, L'art des sons fixés, 1991, Metamkine.

\section{Réinventer le rituel...}

L'idée du concert comme spectacle n'est pas récente, elle est vieille de plusieurs siècles et le public y est naturellement habitué. Ceci dit, le concert sans spectacle, comme l'est le concert acousmatique, devrait ne pas faire de problème et on pourrait très bien il me semble, y accoutumer le public, si les organisateurs et les compositeurs y consacraient plus d'aitention. Je n'ai jamais vu de concert électroacoustique où le problème ait été abordé sérieusement. On prend des salles faites pour le spectacle, avec des scènes éclairées, des fauteuils orientés vers ces scènes, toutes choses qui conditionnent le public à une certaine forme d'attention. Personnellement je trouve stupide de mettre des lumières dans ce genre de concert puisqu'il n'y a rien à voir; cela crée une distraction. Si l'on faisait des concerts dans l'obscurité complète, avec des salles non directionnelles, confortables, etc. le "manque» n'apparaîtrait plus.

Philippe Mion ${ }^{13}$
13. "Le concert, pourquoi? Comment?", Cahiers Recherche/Musique 5 (1977), Paris, INA-GRM, p. 77-78. 
Globalement, il faut tout réinventer dans le rituel des concerts acousmatiques. Questionner les lieux de présentation et bannir les salles à l'italienne où le rapport scène-salle induit un malentendu qui ne se dissipera pas tout au long du concert : malgré eux les auditeurs attendront que quelqu'un vienne. Et qu'il vienne sur la scène, pas dans la salle! Au cours des années, nous avons eu l'occasion de produire des concerts dans des lieux qui n'étaient pas associés au concert traditionnel ${ }^{14}$ et d'une expérience de production à l'autre, nous avons tenté de réinventer le rituel de telle sorte que sur le plan symbolique, l'auditeur se sente en confiance et puisse projeter son imaginaire en commun avec l'interprète, de concert avec lui.

La place de l'interprète dans la salle est à cet égard cruciale. L'aménagement de son espace, la façon qu'il a de s'y rendre, sa manière de se présenter au public, tout cela est à prendre en considération et à mettre au point. On tient trop souvent pour acquis que les choses vont de soi. Notre fréquentation assidue du monde théâtral au cours des dix dernières années nous aura montré que même de la part de gens habitués à la scène, rien n'est laissé au hasard et que même les saluts à la fin d'un spectacle sont mis en scène. II faut apprendre à mettre en scène nos concerts selon de nouvelles règles lou selon des règles anciennes réactualisées) qui doivent être modulées en fonction des dispositifs nouveaux à inventer.

II faut aussi renouveler le rituel afin de proposer au public des points de repère qui le situent. À défaut de cela, celui-ci cherchera les balises habituelles et un malaise s'installera si les conventions ne sont pas respectées. Les concerts de laptop le démontrent à l'envie. Pour une large part constitués de musique enregistrée que l'artiste ne fait que déclencher au début de chaque morceau, ces concerts situent l'interprète exactement là où la musique traditionnelle le fait, c'est-à-dire devant le public et même s'il n'a qu'un rôle minime à jouer, il faut au moins s'assurer qu'il fait semblant de le jover. Mais cette solution, qui n'en est pas une au fond, puisqu'elle ne fait que reproduire le mode de comportement de la musique instrumentale, s'applique mal au concert acousmatique. En effet, l'artiste a besoin d'être dans la salle et non pas sur scène, car il a besoin d'entendre son interprétation dans l'espace et non pas à l'extérieur de celui-ci. II est donc nécessaire de questionner la relation public-interprète, afin de déterminer l'emplacement qui répondra le mieux aux deux nécessités du concert : le rituel public et la qualité de l'interprétation.

Nous croyons quant à nous que cette solution idéale ne sera à notre portée que lorsque nous posséderons notre propre salle de concert dédiée à la présentation de la musique acousmatique. II importe, en effet, que la réflexion qui nous conduira à de nouveaux modes de présentation puisse bénéficier de tout le temps nécessaire sur le plan de la production afin de mettre au point de nouveaux rituels de concerts acousmatiques. En attentant, nous devrons nous contenter de vivre avec les conditions matérielles qui sont les nôtres, c'està-dire frôlant l'indigence presque complète lorsqu'on compare les ressources de l'électroacoustique avec celles de la musique instrumentale ${ }^{15}$.
14. Le planétarium de Montréal en représente certainement l'exemple le plus typique, mais les théâtres La Chapelle et l'Espace GO ou le cinéma Ex-Centris sont autant représentatifs de cet effort de sortir de la salle de concert traditionnelle.

15. Nous avons rédigé un rapport au profit du Conseil des arts du Canada en 2002 et dans lequel il apparaissait que, à la lumière des derniers chiffres publiés, les deux sociétés de concerts électroacoustiques au Canada - I'ACREQ et Réseaux, toutes deux basées à Montréal - ont reçu du CALQ $59000 \$$ pour la saison 1999 2000. Ce qui représente 0,3 de $1 \%$ du budget annuel que le CALQ consacre à la musique... Quant au CAC, la situation n'est guère enviable, puisque dans son dernier rapport annuel (pour l'exercice se terminant le 31 mars 2000) le CAC déclare avoir consacré $21092000 \$$ au secteur musique et quoique ne détaillant pas les montants par organisme, on peut raisonnablement supposer que l'enveloppe globale affectée directement à la production de l'électroacoustique ne dépasse guère les 100 000-150 $000 \$$ ce qui ferait au mieux un pourcentage de 0,7 de 1\%. Marginale, l'électroacoustique, vous avez dit? 


\section{... et raffiner le dispositif}

Auraiton idée de donner à Pollini un piano droit pour l'une de ses rares prestations publiques? Alors pourquoi les systèmes de diffusion sur lesquels on présente cette musique sont-ils à ce point soit déficients sur le plan de la qualité, soit en si mauvais état qu'il ne reste plus rien à en tirer ? Pourtant produire un concert acousmatique aujourd'hui est devenu relativement plus facile qu'autrefois : on trouve de bonnes sonos en location à des prix abordables et l'ensemble des coûts de production d'un concert acousmatique est nettement inférieur à celui d'un ensemble de musique de chambre par exemple ${ }^{16}$. II n'y a donc aucune raison de ne pas faire les choses avec soin, pour le plus grand plaisir des auditeurs. Mais force est de constater qu'il n'y a pratiquement pas d'organismes professionnels qui se consacrent à la promotion de cette musique. Au Canada, deux sociétés de concerts le font et elles sont toutes les deux à Montréal. Aux États-Unis, il n'y en a pratiquement aucune. Et en Europe, en France notamment, la plupart des groupes qui ont déjà défendu cette pratique l'ont à peu près abandonnée au profit du concert mixte ou en direct. Quant au Royaume-Uni, qui est aujourd'hui le centre mondial de l'acousmatique, son réseau de présentation est essentiellement universitaire et malgré le dévouement de ses artisans, il demeure concentré sur les campus pour un public confidentiel.
16. Malheureusement, cet argument se retourne trop souvent contre nous, les subventionneurs en prenant prétexte pour ne distribuer leurs deniers que de façon parcimonieuse au monde électroacoustique, comme si la production de tels événements coûtaient vraiment beaucoup moins chers... Par ailleurs, cela ne les empêche nullement de nous demander d'assurer les mêmes "performances" économiques que nos collègues de la musique instrumentale, pourtant beaucoup plus fortunés.

\section{Seul à seuls}

En poussant encore plus loin la réflexion au sujet du problème posé par la théâtralité du concert en général et de son absence lors du concert acousmatique, on peut se demander si ce dernier n'est pas un cas unique de représentation beaucoup plus proche de celui, intime, de la lecture ou de la radio, que de ceux du théâtre ou du concert instrumental. Peut-être n'y a-til pas de modes de présentation de cette musique en dehors d'un cadre très intime, faisant place à l'intelligence et à l'ouverture d'esprit des auditeurs, venus là en quelque sorte pour se recueillir? Peut-être le concert acousmatique ressemble-t-il davantage au récital de poésie qu'à la performance attendue des arts de la scène? la musique est alors transmise de "seul à seuls" comme l'indiquait Michel Chion dans la citation en tête de cet article, chaque auditeur étant plongé dans sa propre introspection, dans sa propre quête intérieure, ne demandant plus, par conséquent, à être "étonné » par la théôtralité de la représentation mais simplement à être mis en contact avec l'essence même de la musique dans la dernière manifestation du genre où cela est encore possible. Peutêtre. 